\title{
Hypotonic maculopathy secondary to scleral defect in atypical retinochoroidal coloboma
}

\author{
Tito Fiore $^{1}$, Adriana Pellegrino ${ }^{1}$, Marco Lupidi ${ }^{1}$, Alessio Cerquaglia ${ }^{1}$, Fabrizio Giansanti ${ }^{2}$, Carlo Cagini ${ }^{1}$ \\ ${ }^{1}$ Department of Biomedical and Surgical Sciences, Section of Ophthalmology, University of Perugia, S. Maria della Misericordia Hospital, \\ Perugia - Italy \\ ${ }^{2}$ Department of Translational Surgery and Medicine, Eye Clinic, University of Florence, Florence - Italy
}

\begin{abstract}
Purpose: To describe a patient with unilateral hypotonic maculopathy and optic disc edema after spontaneous bulbar perforation of a full-thickness scleral defect.

Methods: An 11-year-old girl underwent scleral buckling surgery. Preoperative and postoperative evaluation included optical coherence tomography and high-resolution 3T magnetic resonance imaging.

Results: The scleral defect was covered with Tenon capsule sheaths and a meridional silicone buckle. One year postoperatively, visual acuity and macular and optic disc morphology were completely restored.

Conclusions: Ocular hypotony related to a defect of the sclera forming the roof of a retinochoroidal coloboma is a rare event. Hypotony was a consequence of a defect at the level of an atypical retinochoroidal coloboma where the scleral wall was found to be absent.
\end{abstract}

Keywords: Retinochoroidal coloboma, Scleral buckle, Scleral defect

\section{Introduction}

We describe a patient who developed hypotonic maculopathy and optic disc edema after spontaneous bulbar perforation related to a coloboma. High-resolution 3T magnetic resonance imaging (MRI) corroborated the diagnosis. The scleral defect was covered both with Tenon capsule sheaths and a meridional silicone buckle. Vision and ocular pressure as well as anatomy were restored during 1 year of follow-up. Hypotony was the consequence of scleral dehiscence at the level of the atypical retinochoroidal coloboma.

\section{Case report}

An 11-year-old girl complained of a 1-month history of painless, blurred vision and visual loss in her right eye (RE). One month earlier, she had been seen for a minor corneal trauma. Her best-corrected visual acuity (BCVA) was 20/200 in the RE

Accepted: June 14, 2016

Published online: July 16, 2016

Corresponding author:

Tito Fiore

Department of Biomedical and Surgical Sciences

Section of Ophthalmology

University of Perugia

S. Maria della Misericordia Hospital

S. Andrea delle Fratte

06156 Perugia, Italy

titofiore@hotmail.com and 20/20 in the left eye (LE). Intraocular pressure (IOP) measured with Goldmann applanation tonometer was $1 \mathrm{~mm} \mathrm{Hg}$ in the RE and $10 \mathrm{~mm} \mathrm{Hg}$ in the LE. The RE anterior segment examination was unremarkable, whereas biomicroscopic fundus examination showed optic disc swelling, blurred optic disc margins, vascular tortuosity, as well as choroidal folds in the macula (Fig. 1A). A choroidal coloboma was noted in the temporal retina (Fig. 1B). The size of the coloboma was about 3 disc diameters. The LE examination was unremarkable.

Optical coherence tomography (OCT) confirmed choroidal folds extending to the macula (Fig. 1E). Orbital imaging with a 3T MRI scanner (Fig. 2) showed decreased axial length of the RE compared to the LE. Anterior chamber and lens were normal in both eyes. Of note, a discontinuity of the scleral tissue was also observed in the RE, and an increased signal due to vitreous prolapse into the discontinuity was appreciated (Fig. 1B).

Based on the clinical examination and supporting imaging studies, a diagnosis of ocular hypotony secondary to spontaneous scleral rupture adjacent to a temporally located retinochoroidal coloboma was made and application of a segmental scleral buckle was planned.

Superior, inferior, and lateral rectus muscles were isolated to expose the sclera. At the site of coloboma, the sclera was absent (Fig. 3A) and vitreous prolapse was noted through bare choroid incompletely covered by Tenon capsule (Fig. 3B). Tenon capsule was adherent to the underlying choroid, therefore any further attempt to separate Tenon capsule from the underlying choroid was abandoned, and both episcleral and parietal sheaths of Tenon (Fig. 3C) were used to cover the coloboma entirely, suturing it beyond its margins. Outside the defect, Tenon capsule was completely separated 


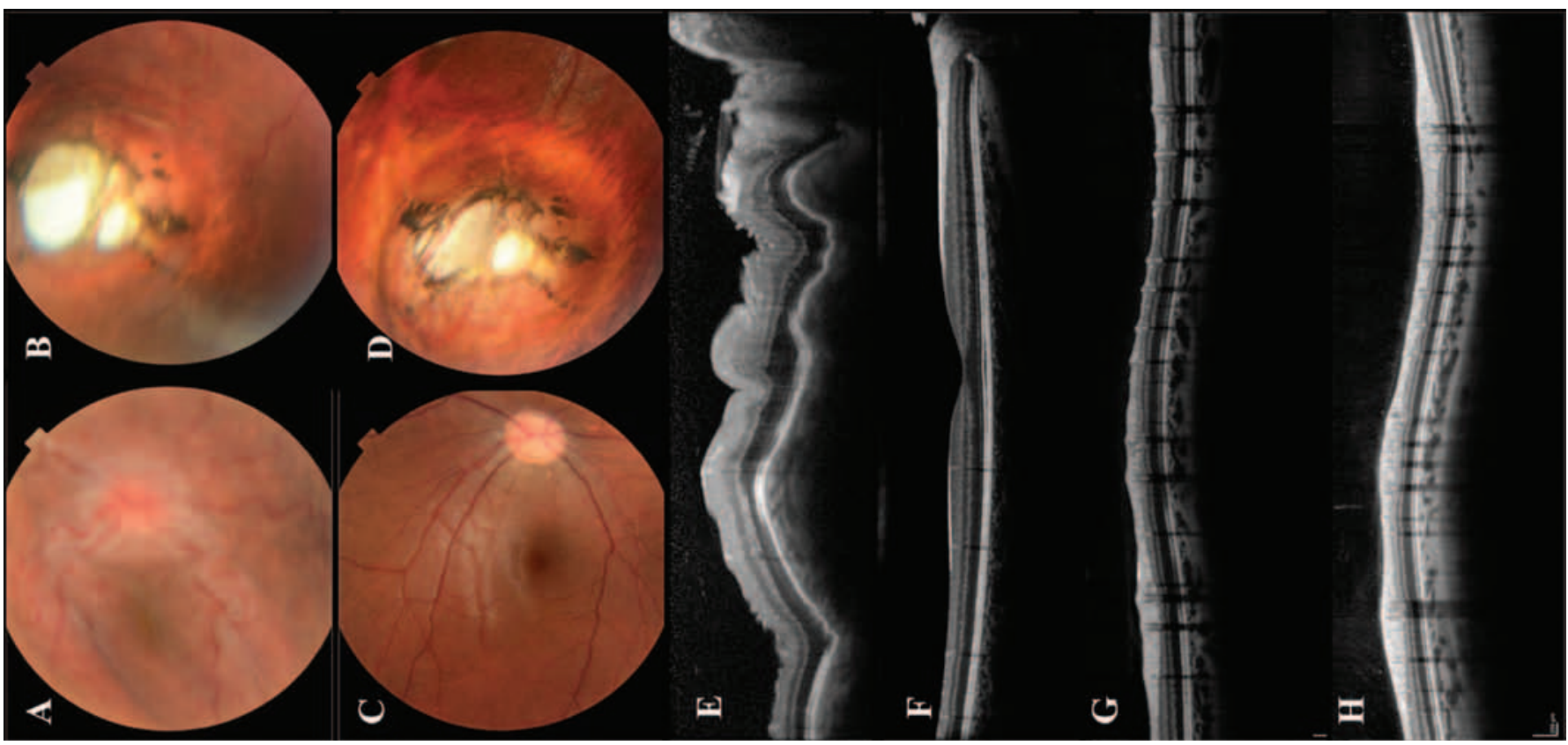

Fig. 1 - (A) Right eye (RE) fundus photographs before surgery show optic disc swelling, blurred optic disc margins, vascular tortuosity, and choroidal folds in the macula, and (B) choroidal coloboma in the temporal retina. (C) The RE fundus photographs 12 months after surgery show the resolution of hypotonic maculopathy and (D) the scleral indentation of coloboma. (E) Optical coherence tomography (OCT) shows choroidal folds in macula before surgery and (F) resolution of macular choroidal folds 12 months after surgery. (G) The OCT of nerve fiber layer (NFL) at the optic disc 12 months after surgery confirms in RE the disappearance of retinal NFL (RNFL) swelling and decreased RNFL thickness compared to the fellow eye $(\mathbf{H})$.
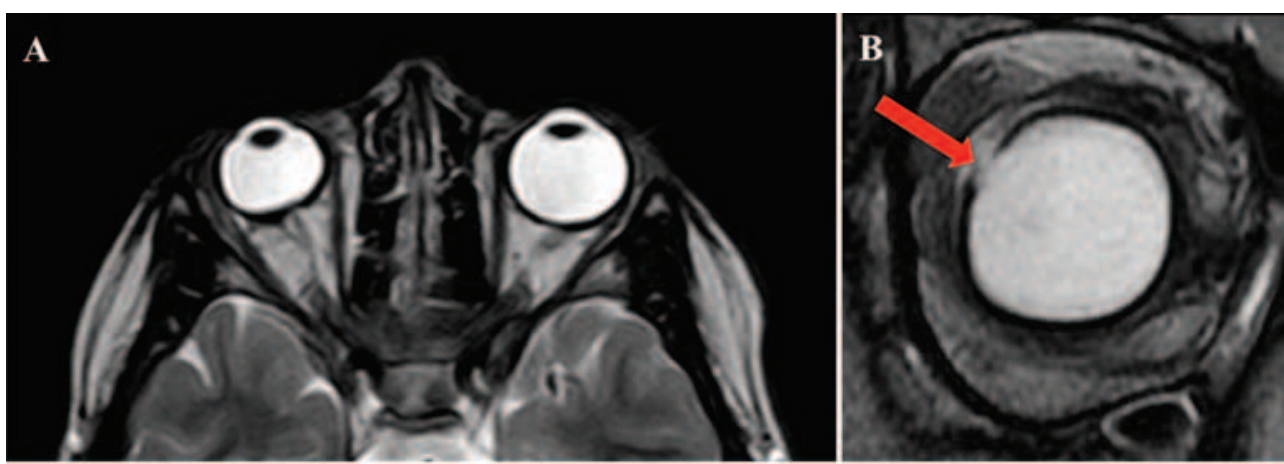

Fig. 2 - (A) Orbital imaging with 3T, axial T2-weighted magnetic resonance imaging (MRI) scan shows asymmetry of the globes. (B) 3T, coronal T2-weighted MRI scan shows vitreous body prolapsing at the level of the scleral discontinuity (red arrow). (C) Postoperative orbital imaging with a $3 \mathrm{~T}$, axial T2-weighted MRI scanner shows symmetry of the eyes and (D) a scleral indentation (green arrow).
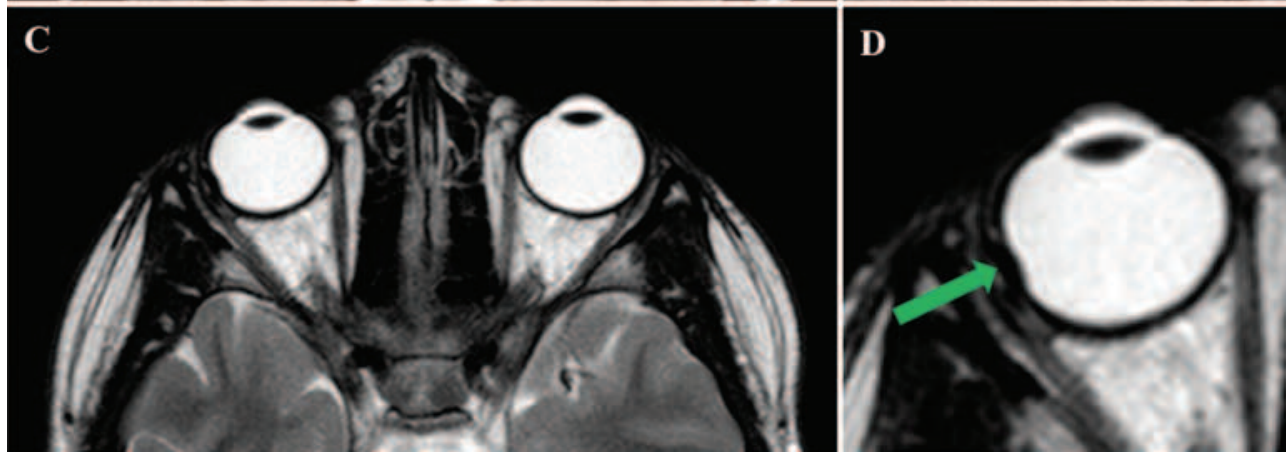

from the sclera and 2 vertical sutures were placed at both sides to fixate a segmental $7.5 \vee 3 \mathrm{~mm}$ solid scleral silicone buckle. The goal was to cover the scleral defect, to seal the filtering site, and to support the prolapsed vitreous by creating a mild indentation (Fig. 3D).
Concomitant with the progressive restoration of foveal contour and decrease of optic disc edema, postoperative BCVA and IOP quickly recovered to $20 / 200$ and $5 \mathrm{~mm} \mathrm{Hg}$ after 2 days, 20/32 and $13 \mathrm{~mm} \mathrm{Hg}$ after 4 weeks, and 20/20 and $11 \mathrm{~mm} \mathrm{Hg}$ after 7 weeks, respectively. One year after surgery, 

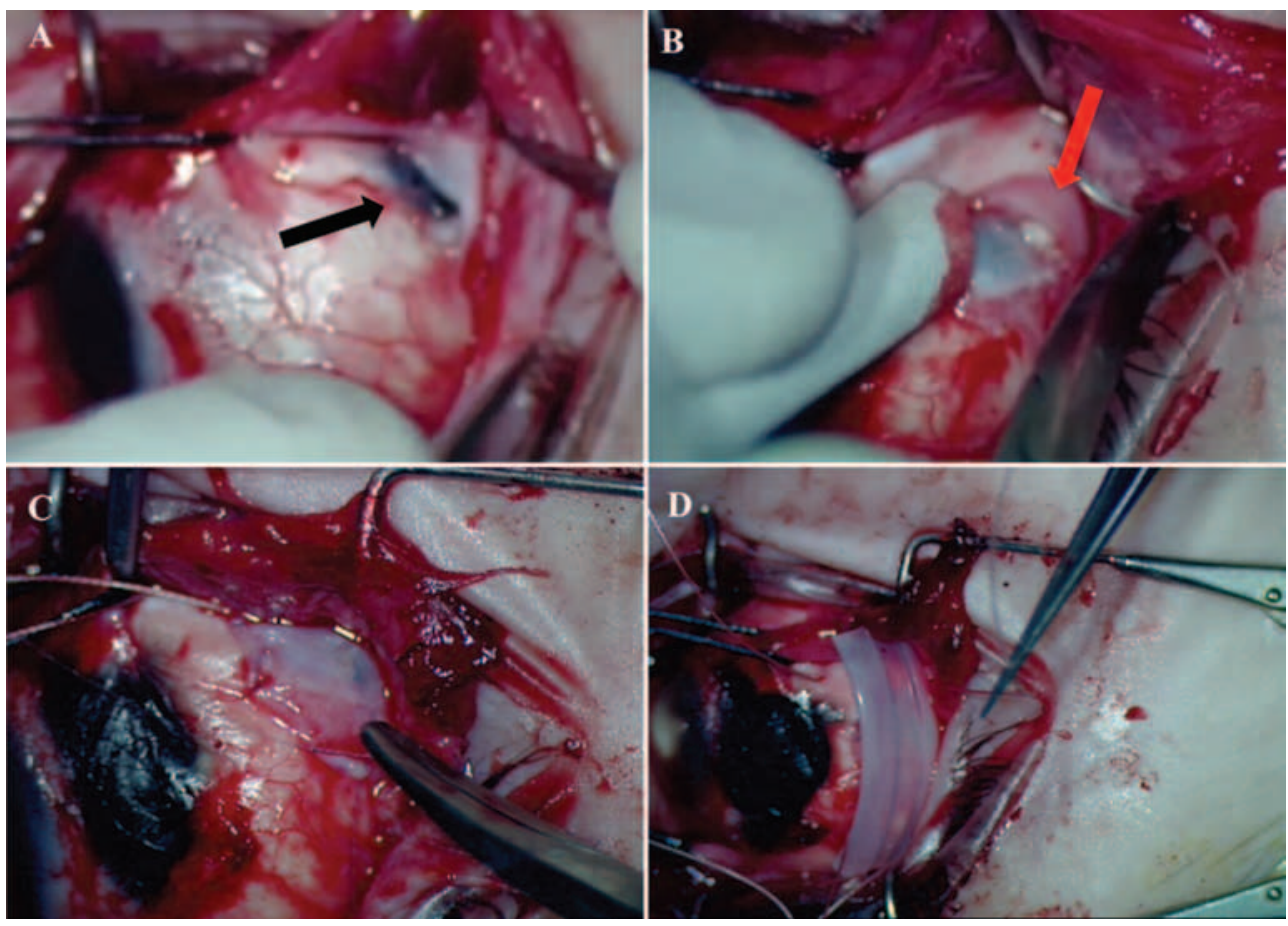

Fig. 3 - Intraoperative color photographs of the right eye (RE) show (A) lack of scleral tissue at the site of coloboma. (B) At the level of the coloboma, vitreous prolapse through the defect was noted (red arrow). (C) Sheaths of Tenon were used to cover the coloboma, and (D) a segmental solid scleral silicone buckle was fixated to cover the coloboma. the patient reported that vision was back to the level before the episode of hypotony. Fundus examination revealed disappearance of optic disc edema, vascular tortuosity, and retinal folds, while a mild scleral indentation at the site of the defect was visible (Fig. 1, C and D). Fundus findings were confirmed on OCT (Fig. 1, F and G). In fact, in the operated eye and in the fellow eye minimal foveal thickness was $223 \mu \mathrm{m}$ and $218 \mu \mathrm{m}$, respectively, whereas at the optic disc, average nerve fiber layer (NFL) thickness was $73 \mu \mathrm{m}$ and $101 \mu \mathrm{m}$, respectively. Postoperative refractive error was $0.75 \mathrm{D}$ of astigmatism compared to a preoperative refractive error of $0.5 \mathrm{D}$. Axial length after treatment was $23.22 \mathrm{~mm}$ in the operated eye and $23.36 \mathrm{~mm}$ in the fellow eye.

\section{Discussion}

Choroidal coloboma is a rare ocular malformation related to defective closure of the embryonic fissure typically located inferonasally, which normally occurs on day 33 of fetal development. The reported incidence of retinochoroidal coloboma is $0.14 \%$, and $40 \%$ of patients may develop a rhegmatogenous retinal detachment sometime during their life (1). Few cases of spontaneous bulbar perforation of congenital coloboma have been reported in the literature (2-7).

This case represents what we believe to be the first reported case of a coloboma associated with hypotonous maculopathy in an 11-year-old girl. Of note, in our patient the lesion was temporal, making the coloboma atypical. There was no history of trauma or necrotizing scleritis. In agreement with previous reports on adult patients, optic disc edema, choroidal folds, and vascular tortuosity gradually regressed after the IOP was surgically restored, but different from a previous report (4), disappearance of optic disc edema was observed, possibly followed by a mild NFL thickness decrease. Another peculiarity that has never been reported in such a case was that Tenon capsule was strongly adherent to the underlying bare choroid, making any surgical maneuver to separate Tenon capsule a high risk for bleeding. For this reason and with the intent to completely cover the coloboma before adding a buckle, Tenon sheaths were sutured to the sclera, potentially contributing to the sealing of the scleral defect along with the segmental buckle. Whether the strong adherence between choroid and Tenon capsule was due to the young age, to the posterior location of coloboma, or to the extent of scleral defect is unknown, but supported by developmental studies.

Certainly in our case, the use of lyophilized homologous dura mater (2) glued to the affected portion would have been barely possible because of the difficulty to properly expose the affected area. Alternatively, suture imbrication (5) of the scleral defect would have been difficult because of the posterior location and large size. Finally, given the high incidence of retinal detachment related to choroidal coloboma (1), any attempt to simply cover bare choroid with a patch of polytetrafluoroethylene (6) or to seal the sclera with a retrobulbar injection of autologous blood (7) were avoided. The bare choroid was covered by sheets of Tenon capsule, while the use of a $7.33 \mathrm{~mm}$ segmental meridional buckle allowed us to firmly close the scleral defect with little effect on refraction. This represented a prophylactic approach to prevent a future retinal detachment related to retinal break formation within the coloboma, as previously reported (1). Given the absence of retinal break in the intercalary membrane, laser photocoagulation along the border of coloboma (4) was also avoided.

Concerning the origin of the hypotony, it was only during the surgery that the hypothesized scleral rupture was effectively identified. Therefore hypotony should be considered as 
the consequence of a dehiscence at the level of retinochoroidal coloboma where the scleral wall was absent, instead of a consequence of scleral rupture (4-7) over an area of scleral thinning (4) or scleral ectasia (5).

In conclusion, ocular hypotony related to a defect of the sclera in the roof of a temporally located retinochoroidal coloboma is a rare event, and this entity has not been reported previously in young patients. The surgical treatment to cover the scleral defect suturing sheaths of Tenon capsule with indenting scleral buckle was a technique to correct the cause of hypotony, to restore the anatomy of the macula and its function, and to prevent postoperative complications such as persistent hypotonic maculopathy, endophthalmitis, and retinal detachment.

\section{Disclosures}

Financial support: No financial support was received for this submission.

Conflict of interest: None of the authors has conflict of interest with this submission.

Meeting presentation: The manuscript was presented at STUEMO 2015.

\section{References}

1. Pattern of retinal breaks and retinal detachments in eyes with choroidal coloboma. Gopal L, Badrinath SS, Sharma T, Parikh SN, Biswas J. Ophthalmology. 1995;102(8):1212-7.

2. Mori S, Komatsu H, Watari H. Spontaneous posterior bulbar perforation of congenital scleral coloboma and its surgical treatment: a case report. Ophthalmic Surg. 1985;16(7): 433-436.

3. Gupta A, Narang S, Gupta V, Sharma A, Pandav SS, Singh P. Successful closure of spontaneous scleral fistula in retinochoroidal coloboma. Arch Ophthalmol. 2001;119(8):1220-1221.

4. Viola F, Morescalchi F, Gandolfo E, Staurenghi G. Ocular hypotony secondary to spontaneously ruptured sclera in choroidal coloboma. Arch Ophthalmol. 2004;122(10):1549-1551.

5. Fine HF, Sorenson JJ, Spaide RF, Cooney MJ, Fisher YL, Del Priore LV. Spontaneous scleral rupture adjacent to retinochoroidal coloboma. Retin Cases Brief Rep. 2008;2(4):296-298.

6. Gaucher D, Ballonzoli L, Saleh M, Sauer A, Bourcier T, SpeegSchatz C. [Spontaneous scleral rupture revealed by hypotony maculopathy]. J Fr Ophtalmol. 2009;32(6):438.e1-438.e6.

7. Mahr MA, Garrity JA, Robertson DM, Herman DC. Ocular hypotony secondary to spontaneously ruptured posterior staphyloma. Arch Ophthalmol. 2003;121(1):122-124. 